\title{
La Legación Diplomática Chilena en los Estados Unidos de Colombia (1879)*
}

\author{
Chilean Embassy in the United State of Colombia (1879)
}

\author{
Marcelo Jara Román \\ Felipe López Pérez ${ }^{* * *}$ \\ Universidad Católica Santísima de Concepción, Concepción, Chile
}

Recibido: 30 de abril de 2013. Aprobado: 13 de octubre de 2013.

\begin{abstract}
Resumen
El presente trabajo tiene por objeto determinar, a través del análisis de los archivos de la Legación de Chile en los Estados Unidos de Colombia, la posición de los gobiernos chileno y colombiano, frente a la neutralidad del istmo de Panamá y el eventual tráfico de armas de Perú por dicha vía en 1879, además, establecer las acciones y logros de la misión diplomática chilena en el país caribeño durante 1879. Algunos hitos denunciados por el encargado de Negocios en Colombia, Francisco Valdés Vergara, pueden ser leídos - desde la óptica del equilibrio de poder- como un concreto desafío a la soberanía y los intereses de Chile en la Guerra del Pacífico por parte de la unión colombiana.
\end{abstract}

Palabras claves: diplomacia, equilibrio de poder, Guerra del Pacífico

\begin{abstract}
The objective of this article is to study the files of the Chilean embassy in the United States of Colombia, in order to determine the position of the Chilean and Colombian govern-

Este trabajo formó parte del proyecto de investigación interno de la Universidad Católica de la Santísima Concepción DIN/09, titulado: "Guerra, diplomacia y opinión pública: las relaciones internacionales entre Chile y Colombia durante la guerra y posguerra del Pacífico, 1879-1886”.

** Universidad Católica de la Santísima Concepción, Facultad de Comunicación, Historia y Ciencias Sociales, Concepción, Chile. Correo electrónico: mjara@ucsc.cl

*** Universidad Católica de la Santísima Concepción, Facultad de Comunicación, Historia y Ciencias Sociales. Concepción, Chile. Correo electrónico: flopez@ucsc.cl
\end{abstract}


ments regarding the neutrality of the Isthmus of Panama and the eventual weapons traffic through that way to Peru in 1879. Additionally this work tries to establish the actions and achievements fulfilled by the Chilean mission before that Caribbean country during 1879. Some of those achievements, registered by Francisco Valdés Vergara, ambassador in Colombia, can be understood - from the Balance of Power's view- as a clear challenge posed by Colombia to the sovereignty and interests of Chile, during the Pacific War.

Keywords: diplomacy, Pacific War, balance of power

\section{Introducción: la política exterior de Chile. La idea del equilibrio de poder}

La historiografía que trabaja la historia de la Guerra del Pacífico como eje axial y como temática central de sus relatos, ha dilucidado ciertas características en el ámbito de las relaciones exteriores de Chile durante el siglo XIX. Períodos que se vinculan al crecimiento económico y a la estrecha relación con potencias foráneas, tanto en la introducción de capital, como de tecnologías en la extracción de materias primas, así también en la emergencia de un precario mercado común (Sater, 1981, 1986, 1990, 2007). En el imaginario colectivo de la oligarquía, la consolidación de la institucionalidad se puede entender a través de la política interna y externa que Chile desarrolló en el tiempo sindicado (Góngora, 2006; Salazar, 2005; Collier, 2005). Esto puede entenderse en base al concepto de seguridad y su materialización en la idea del equilibrio de poder entre las naciones sudamericanas, elemento indispensable para el desarrollo sostenido de los intereses económicos y hegemónicos de la elite decimonónica (Hilton, 2001; Burr, 1967). Dicho de otro modo, fue una política de contención ajustada a la medida de fuerzas, poder y capacidad de maniobra de una potencia extra o intracontinental (Fermandois, 2005; Rubilar, 2010; Burr, 1967).

Frente a la posición planteada, ¿qué se entiende por equilibrio de poder? Según Neila (2008), "es el principio que aspiraba a las acciones políticas, diplomáticas y militares orientadas a preservar un determinado equilibrio territorial y político entre los estados y evitar el predominio de alguno de ellos" (p. 347).

El concepto anteriormente consignado, dada la complejidad de las relaciones internacionales y su estudio, puede ser entendido como una política, objeto político o un intento deliberado por prevenir un poder predominante (Barbé, 1987); así también —siguiendo a Neila (2008) - como un "sistema internacional fundamentado en la naturaleza interestatal del mismo" (p. 347). En tanto, para Burr (1957, 1967), el equilibrio de poder 
es la compensación de fuerzas entre un grupo de naciones soberanas, que evitan la emergencia de un poder superior dentro de ellas. Esto significa prevenir una hegemonía y la voluntad de un tercero que amenaza los objetivos intra y supranacionales de un determinado país, y su relación con sus pares. Asimismo, de acuerdo a Meneses $(1986,1989)$, el equilibrio se ha visto mermado por el desigual desarrollo dentro de algunos factores como la población, el desarrollo económico y tecnológico, estabilidad política y poder militar.

Durante el siglo XIX, según Burr (1967) y Rubilar (2010), la maduración del concepto de poder entre las naciones latinoamericanas, debía cumplir tres condiciones básicas. En primer lugar, las naciones debían tener un mínimo esencial de soberanía, tales como límites territoriales definidos y gobiernos efectivos. En segundo término, que las relaciones entre ellos tenían que estar sujetas a un estándar de influencia no continental, y, finalmente, que los canales de comunicación y los puntos de contacto entre las naciones latinoamericanas debían estar lo suficientemente desarrollados, como para poder hacer que cada país fuera consciente de sus intereses y de cómo estos se verían afectados por la acción e intervención de otros.

Dichas condiciones fueron adquiridas lentamente por los países latinoamericanos. De esta forma, se determinó — durante la primera mitad del siglo XIX — su posición de poder en el sistema internacional americano. En el segundo tramo secular, adquirió en esta lógica y comprensión del equilibrio de poder- una vital importancia el factor Fuerzas Armadas, en especial, la Armada. Según Rubilar (2010), esta última cumplió la misión de proteger los objetivos e independencia nacional. "Lo anterior unido a los medios políticos y diplomáticos que el Estado utiliza, con el fin de reorientar la política internacional de cualquier poder regional que pudiera amenazar el equilibrio entre el continente" (p. 4).

Esta política de contención de la diplomacia chilena hacia sus pares latinoamericanos antes de la Guerra del Pacífico, se puede vincular con la relación de neutralidad de los Estados Unidos de Colombia y su carácter de no interventor en los asuntos limítrofes y económicos de sus vecinos sureños (Rivas, 1961). En efecto, cabe señalar la importancia del estudio de las relaciones internacionales como un fenómeno vinculado, en un primer estadio, con el desarrollo interno de las sociedades en sus múltiples facetas y procesos, y en un segundo acápite, con la política mundial en la que se halla inserta (Fermandois, 2005; Pereira, 2009). 


\section{Estado de la cuestión}

Respecto a las relaciones chileno-colombianas — dos países de la zona andina del Cono Sur y que tuvieron, durante el período reseñado, una fuerte suspicacia mutua por el dominio de la franja costera del Pacífico-, un autor ha publicado, en varios artículos especializados, la vinculación existente entre estos dos Estados, dándole énfasis a la amistad-hostilidad provocada por el irregular comportamiento de la nación andina en la época de la Guerra del Pacífico y posterior a esta. Rubilar (2008) señala que los fundamentos de la rivalidad patente entre estos dos gobiernos, se debió a la filiación histórica que tuvo Colombia con Estados Unidos en el siglo XIX, y la inestable soberanía que tuvo esta confederación sudamericana en algunas latitudes de sus territorios, como Panamá, por ejemplo, además de la creciente polarización ideológica entre los bandos conservadores y liberales por el control de las leyes, la propiedad latifundista y el poder.

Un hito que rompió esta odiosidad entre Chile y Colombia, fue la misión diplomática (1880-1886) del poeta chileno José Antonio Soffia que, por un lado, calmó la elevada temperatura de una casi inclusión de Colombia como un actor más de la guerra de 1879; y, por otro, a relevar al incendiario ministro plenipotenciario de Chile en Colombia, Francisco Valdés Vergara, y su afanado trabajo como protector de los intereses chilenos en el istmo y la neutralidad de este en el conflicto (Rubilar, 2010; López, 2011; Jara y López, 2012).

Sobre este último punto, los trabajos historiográficos clásicos de Vicuña Mackenna (1881, 1880), Bulnes $(1911,1914)$, Barros Arana (1914), y los de Barros van Buren (1990), Burr $(1967)$ y Sater $(2007,1990,1986,1981)$ no dedican demasiadas líneas a la gestión de la diplomacia chilena en Colombia durante la Guerra del Pacífico. Solo detallan, dentro del contexto de las campañas y estrategias de la ofensiva chilena, peruana y boliviana, el tráfico de armas desde el istmo de Panamá y las consecuencias que esto tuvo en el desarrollo de las operaciones armadas. De las reclamaciones del cónsul chileno en Panamá hay pocas menciones, al igual que el trabajo de Valdés Vergara (Jara y López, 2012).

Por una parte, siguiendo la lógica del equilibrio de poder y el factor naval-militar como dos elementos de orden en el escenario sudamericano; la influencia de la política exterior de las grandes potencias en la configuración de la política interna y externa de las naciones pequeñas o satélites, y la idea del uso de la diplomacia en caso de una amenaza de los intereses nacionales y la soberanía de un Estado, este estudio se propone responder las siguientes preguntas: ¿cómo se expresó epistolarmente la posición de los gobiernos chileno y colombiano, frente a la neutralidad del istmo de Panamá y el eventual tráfico

\section{Si Somos Americanos. Revista de Estudios Transfronterizos}


de armas de Perú por dicha vía? ¿Cuáles fueron los principales logros y acciones de la Legación de Chile en los Estados Unidos de Colombia durante 1879?

En correlación con el párrafo precedente, se plantean para esta investigación los siguientes objetivos. El primero de ellos es determinar, a través del análisis de los archivos de la Legación de Chile en Colombia, la posición de los gobiernos chileno y colombiano, frente a la neutralidad del istmo de Panamá y el eventual tráfico de armas de Perú por dicha vía en 1879. Mientras que el segundo intenta establecer las acciones y logros de la Legación de Chile en los Estados Unidos de Colombia durante 1879.

Este trabajo, además, se sostiene sobre la siguiente hipótesis de estudio: las relaciones internacionales de Chile y Colombia durante el período sindicado (1879) fueron tensas, aun cuando se mantuvieron - desde el punto de vista diplomático- las misiones y legaciones en ambos países. En el curso de la gestión de Valdés Vergara (1879-1880) sucedieron algunos hitos que pueden ser leídos como un concreto desafío a la soberanía y los intereses de Chile en la guerra por parte de Colombia, como, por ejemplo, la permisividad en el tráfico de pertrechos por el istmo, así como también la manifiesta simpatía del presidente de dicho país, Julián Trujillo, por la causa peruano-boliviana.

\section{Los principales problemas diplomáticos según la Legación de Chile en Panamá y Colombia}

En la revisión de los archivos de la Legación de Colombia en Chile y viceversa, el secretario de la Legación ésta y Relaciones Exteriores del país caribeño al inicio de la Guerra del Pacífico ${ }^{1}$, Manuel Ancízar², indicó en Bogotá, el 15 de abril de 1879, que dada la política de buenos oficios y amistad con las naciones en pugna, era necesario someter a arbitraje la disputa económica y territorial que enemistaba a Chile con Bolivia y Perú3.

La declaración de guerra de Chile a Perú y Bolivia fue el 5 de abril de 1879.

2 Manuel Ancízar nació en la hacienda El Tintal, Fontibón (Cundinamarca), el 25 de diciembre de 1812, y falleció, en Bogotá, el 21 de mayo de 1882. Fue un abogado, escritor, político, profesor y periodista colombiano. En su labor como profesional de las comunicaciones, fundó el periódico El Neogranadino. Colaborador de El tiempo, El Correo, El Siglo, El Liberal y el Repertorio de Venezuela, y de El Museo de Santiago de Chile. Además, fue el cuñado del literato José María Samper, ministro plenipotenciario de Colombia en Chile (1884). Para mayor referencia, véase a Rodríguez-Arenas (2006, pp. 65-67).

3 Bogotá siguió al pie de la letra el artículo 11 del Tratado Mosquera-Irarrázaval de 1844, donde se establecía la neutralidad en caso de que uno los contrayentes entrara o tuviera un conflicto con un tercero. Se copia un fragmento de dicho texto: "Ninguna de las partes contratantes franqueará auxilios de ninguna clase a los enemigos de la otra, con el objeto de facilitar las operaciones de la guerra; antes por el contrario empleará sus buenos 
A partir de este ofrecimiento de mediación, Colombia, en tanto, se mantuvo epistolar y diplomáticamente en una posición de observador. En un oficio enviado por el titular del Ministerio de Relaciones Exteriores de Colombia, Luis Carlos Rico, a su homólogo chileno el 16 de junio de 1879, el gobierno colombiano lamentaba que, definitivamente, a pesar de las campañas marítimas del conflicto del Pacífico, se haya roto la paz entre los Estados en pugna y que tenían una vinculación de amistad con Colombia (Fondo Ministerio de Relaciones Exteriores de Chile, 1877-1886, vol. 181, s.f).

Un mes más tarde, Rico respondió a la continua interrogación que hizo Chile al gobierno colombiano respecto a la neutralidad del Estado Soberano de Panamá —miembro de los Estados Unidos de Colombia- en el conflicto del Pacífico. Asimismo, la preocupación del presidente colombiano, Julián Trujillo ${ }^{4}$, en una misiva enviada a su par, Aníbal Pinto Garmendia ${ }^{5}$, indicaba que su menester era que Chile y Bolivia arreglaran sus diferencias. Por esa razón, Trujillo envió a Chile al ministro plenipotenciario Pablo Arosemena -hábil político panameño y futuro presidente del Istmo (1910-1912) -, quien siguiendo las disposiciones de los tratados vigentes entre ambos países, se dispuso a ofrecer los "buenos oficios" y un posible arbitraje (mediación) para el establecimiento de la paz entre ambas naciones en conflicto ${ }^{6}$.

A Chile le interesaba mantener buenas relaciones con los otros países de Sudamérica que no se encontraban en una posición parcial con la entente boliviana-peruana. En efecto, en un oficio enviado por el ministro de Relaciones Exteriores de Chile, Domingo Santa María González, a su par en Colombia, nuestro país manifestó una clara queja

por motivo de la conducta observada por el Presidente del Estado soberano de Panamá en relación con el tránsito de elementos de guerra por el istmo, conducta que V.E. estima como violatoria del tratado de amistad, comercio i navegación que existe entre

oficios, i si fuese necesario, su mediación, para el establecimiento de la paz; no permitiendo la entrada en sus puertos i costas, a los corsarios enemigos, ni a las presas que estos hicieren a los ciudadanos o comerciantes de la Nueva Granada o Chile" (De Plaza, 1850, p. 262).

4 Julián Trujillo Largacha fue un estadista, abogado, político y militar colombiano. Nació en Popayán, Cauca, el 28 de enero de 1828, y falleció, en Bogotá, el 18 de julio de 1883. Fue presidente de Colombia durante el período 1878-1880. Véase a Arteaga, Manuel y Arteaga, Jaime (1986).

5 Aníbal Pinto Garmendia, nació en Santiago, el 15 de marzo de 1825 y falleció en Valparaíso, el 9 de junio de 1884. Fue abogado, político y presidente de Chile. Antes de asumir la Presidencia de Chile, perteneció al Partido Liberal y desempeñó diversos cargos diplomáticos y ministeriales (Bizzarro, 2005, pp. 585-586).

$6 \quad$ Los antecedentes que hicieron posible esta negociación bilateral, datan de los acuerdos suscritos en 1822 (Tratado Mosquera-Echeverría), 1844 (Tratado Mosquera-Irarrázaval) y 1853 (Convención Consular Varas-Ancízar). Véase a Rivas (1961) y Barros van Buren (1990).

106 Si Somos Americanos. Revista de Estudios Transfronterizos 
Colombia i Chile (Fondo Ministerio de Relaciones Exteriores de Chile, 1877-1886, Vol. 181, s.f.).

Frente a lo señalado por el ministro Santa María, Bogotá se comprometió a respe$\operatorname{tar}$ fehacientemente la neutralidad ${ }^{7}$ en el conflicto entre Chile, Bolivia y Perú. No obstante, la posición de Colombia se vio mermada por la irregular conducta de Panamá, Estado soberano dependiente administrativamente de Colombia a partir de 1841. Lo interesante de esta situación radicó en los asuntos no resueltos entre Panamá, Colombia y Estados Unidos, generados por el parcial tratado Herrán-Cass (1857), donde prácticamente Nueva Granada ${ }^{8}$ tuvo que indemnizar al país del norte. Este incidente, conocido como "la tajada de sandía" (Souler 1999, 1987), rememoró el intervencionismo norteamericano expreso en el tratado Mallarino-Bidlack de 1846, donde Estados Unidos adquirió, entre otros, franquicias y exenciones tributarias, de cabotaje y de comunicación, en todos los puertos del país caribeño.

A pesar del dilecto comportamiento de la diplomacia colombiana y su atenta disposición a responder las inquietudes chilenas, a fines de mayo de 1879, el diplomático chileno Domingo Godoy Cruz fue apresado en el puerto del Callao cuando se dirigía a Colombia a cumplir sus labores como ministro plenipotenciario. Este hecho dificultó las relaciones entre Chile, la entente peruano-boliviana y Colombia. Sin embargo, la cancillería bogotana remitió el siguiente informe a sus pares peruanos:

El poder ejecutivo de la unión (colombiana) ha visto con pena este suceso que lo ha privado del emisario de una nación amiga e interpone en todo momento los buenos oficios para interceder ante V. E. el presidente de la república peruana, con el propósito de que sirva poner en libertad a Godoy y no se le impida la continuación de su viaje (Boletín de la Guerra del Pacífico, 1979, p. 190).

En este sentido, la preocupación del gobierno colombiano se dividió en dos polos. En primer lugar, en solicitar a Perú el arribo inmediato del plenipotenciario de Chile, apresado en el Callao, a Colombia, y en segundo, en dilucidar la neutralidad del istmo en la guerra. Frente a este último hecho, Luis Carlos Rico manifestó que el Estado de Panamá no negaba el tránsito de armas y demás elementos de guerra por el ferrocarril

7 Este principio de libre tránsito del ferrocarril se ratificó en la comunicación enviada por el ministro de Relaciones Exteriores de Colombia, Luis Carlos Rico, a su homólogo chileno, Domingo Santa María, el 18 de julio de 1879 (Fondo Ministerio de Relaciones Exteriores, 1877-1886, vol. 181).

8 El período comprendido entre 1830-1862 se conoce como el tiempo de la República Neogranadina. Desde 18631886 es la era de los Estados Unidos de Colombia (Arteaga y Arteaga, 1986). 
interoceánico, sin embargo, y así lo consideran los tratados firmados con Chile, Estados Unidos, Perú y Bolivia:

El camino de carriles de hierro entre el Atlántico i el Pacífico en el istmo de Panamá ha sido declarado vía de tránsito enteramente franca para el comercio universal; liberalidad que implica la exoneración del deber de averiguar el orijen, clase i destino de las mercaderías que por allá pasen.

No habiendo aduanas en las puertas de Colón i Panamá, es impracticable la fiscalización sobre toda la carga que le transporta del uno al otro mar i sería de todas luces inconveniente la mui defectuosa que se pretendiera establecer.

En este supuesto sería preciso permitir el tránsito de elementos de guerra en su calidad de artículos de comercio siempre que se manifestasen como enviados a puertos neutrales de cualquiera de los países litorales del pacífico, lo cual daría lugar a un tráfico que podría favorecer momentáneamente a uno de los beligerantes (Fondo Ministerio de Relaciones Exteriores de Chile, 1877-1886, vol. 181, s.f.).

En consecuencia, por el manifiesto libre tránsito de mercaderías por el ferrocarril de Panamá, la Cancillería colombiana resolvió no atender stricto sensu la solicitud de la diplomacia chilena. Ante esto, Chile no quiso dejar impune la actitud de Colombia respecto a la cuestión del tráfico de armas. Por esta razón, Domingo Santa María, ministro de Relaciones Exteriores de Chile, dirigió la siguiente minuta a los representantes nacionales en dicho país:

La conducta de la autoridad colombiana, negándose con frívolas escusas, a atender la solicitud que le hacía nuestro cónsul para que impidiera el embarque de elementos bélicos destinados a Bolivia i el Perú, elementos cuya existencia no podía allí ponerse en duda, ha causado en mi Gobierno una penosa impresión. Estábamos muy lejos de aguantar que los agentes del poder público de Colombia, que en toda ocasión ha mantenido con Chile relaciones de constante i leal amistad, pudieran faltar, en daño de nuestro país, a los deberes que una severa neutralidad les impone (Archivo General del Ministerio de Relaciones Exteriores de Chile, 1879-1881, vol. 62A, fojas 12-13).

Entonces, la reacción del gobierno chileno de mantener una política exterior férrea (Ministerio de Relaciones Exteriores de Chile, 1879, p. 273), clara y precisa, pero por 
sobre todo rigurosa, se materializó con la llegada de Francisco Valdés Vergara9 ${ }^{9}$ quien asumió en reemplazo del cautivo Godoy.

\section{Colombia comunica la neutralidad del istmo}

La posición del gobierno colombiano, independiente de las denuncias permanentes del cónsul chileno en el Estado de Panamá, Antonio Jiménez, y del encargado de Negocios nacional en Colombia, Francisco Valdés Vergara, manifestó epistolarmente una posición neutral en el conflicto y una política exterior de buenos oficios.

Según Rivas (1961), las acusaciones hechas por Vergara, no tenían fundamento alguno, ya que siendo el Istmo un lugar obligado de tránsito para las armas y municiones para los beligerantes del Pacífico, la administración Trujillo dictó, a insinuación del Presidente de Panamá, una resolución (2 de junio de 1879) en la cual se manifestó que el ferrocarril del Istmo y dicha zona fue declarada "vía de tránsito enteramente franca para el comercio universal, liberalidad que implicaba la exoneración del deber de averiguar el origen, clase y destino de las mercaderías que por ella pasaran" (p. 478).

No obstante, la Cancillería chilena intentó conservar la amistad entre ambos países y evitar que Colombia se convirtiera en un tertius gaudens o un pívot que beneficiara los intereses de una potencia foránea. El testimonio de los diplomáticos —en un primer estadio-constituyó un elemento probatorio que podría quebrar las relaciones entre Santiago y Bogotá. Sin embargo, en las misivas ulteriores se verá que Colombia y Chile son víctimas y actores secundarios del complot ideado por Estados Unidos ${ }^{10}$ para solucionar

9 El ministro de Relaciones Exteriores de Colombia, Luis Carlos Rico, en oficio despachado el 28 de agosto de 1879, indica que "por el atento despacho de V. E. fechado el 25 de junio último, se impuso el ciudadano presidente de la unión de que el gobierno de esa república había tenido a bien acreditar al honorable señor Francisco Valdés Vergara con el carácter de su encargado de negocios por haber sido detenido en el Callao por autoridades peruanas, el señor Domingo Godoy, que tenía esa misión" (Fondo Ministerio de Relaciones Exteriores, 18771886, vol. 181, s.f.). Además, es esta misiva se reconoce la legitimidad de Valdés en su cargo por parte del Poder Ejecutivo colombiano. Véase también documentación entre Domingo Gana y Jorge Huneeus, 25 de junio de 1879 (Fondo Misiones Diplomáticas, 1879a, vol. 25. s.f.).

10 Estados Unidos no solo se comprometió a resolver diplomáticamente el conflicto entre Chile, Perú y Bolivia, sino también contribuyó con armamento de contrabando. El periódico panameño "La Estrella", edición del 13 de septiembre de 1879, dio cuenta de tal hecho. Copiamos un extracto de tal información: "Ocho carros de armas que (el barco) el Limeña dejó aquí en su último viaje i que no las tomó por falta de tiempo; cuatrocientas pipas venidas de Nueva York con la marca J. B. E. por vapor Acapulco destinadas a la casa Grace Brother al del Callao [...]; seiscientos setentas i cinco cajones de capsulas marcados 'P. D', que pesan ciento cincuenta i tres mil letras i cinco cajones de armas traídos de Nueva York para el vapor americano Crescent City; todo lo cual está ya 
el conflicto del Pacífico, evitando la ruina económica de Perú, la pérdida de soberanía de los territorios en disputa y la ruptura del equilibrio de poder en Sudamérica (Burr, 1967).

Tanto los oficios enviados como los recibidos por la Legación Chilena en los Estados Unidos de Colombia -durante la primera quincena de septiembre- tuvieron el tenor de felicitar a Chile por la celebración de las fiestas patrias, el 18 y 19 del noveno mes del año a pesar de la guerra. Lo anterior, vino a fortalecer el espíritu patriótico y nacionalista de Chile, además de la política de buenos oficios y de mediación de parte de las potencias europeas como Alemania o Dinamarca (Fondo Misiones Diplomáticas, 1879b, vol. 25, s.f.). Sin embargo, las epístolas con noticias frescas del tráfico de armas en el Istmo no pararon. El cónsul nacional en Panamá, Antonio Jiménez, indicó, el 19 de septiembre de $1879^{11}$, que el transporte peruano Oroya ${ }^{12}$ había fondeado al frente de la isla Flamenco, en la costa pacífica de Panamá. Las misión que tenía esta embarcación, según indica Farcau (2000), era cargar desde el puerto del istmo, "dos torpederas Herresford, cuatro mil rifles, tres millones de cartuchos de fusil, seis cañones Krupp y cuarenta escudos" (p. 80), mercancía proveniente de Honduras y Costa Rica.

En efecto, la respuesta del secretario de Gobierno del Estado Federal del istmo, José María Alemán, fue inmediata. El político panameño indicó que:

se han dictado por el poder ejecutivo las órdenes del caso para impedir el embarque a los elementos de guerra de que trata su estado comunicación, lo mismo que se ha verificado, con el mismo fin en las veces anteriores que a este respecto se ha dirigido a ud. mi despacho. Es por orden del ciudadano, presidente del Estado, que doy a ud. respuesta en los anteriores términos a la nota de ud. antes citada (Fondo Ministerio de Relaciones Exteriores, 1879-1886, vol. 217, foja 18).

embarcado en la lancha número uno de la compañía del ferrocarril; I algunos bultos entre los que se encuentran torpedos formidables venidos de Nueva York por el vapor Edén, al cuidado del coronel peruano, señor Lara, quien trae además dos ingenieros" (Fondo Ministerio de Relaciones Exteriores, vol. 217, fojas 11-12).

11 De hecho, Jiménez, en una misiva enviada a José Alemán, secretario de Gobierno del Estado Federal del Istmo, indica que: "Hai en este puerto un inmenso cargamento bélico, parte en tierra, parte en una de las lanchas del ferrocarril y es de esperarse, con razón sobrada que el Oroya venga a recibir a su bordo y conducir al Perú, el cargamento a que me he referido. De igual forma, es de conocimiento público que vecinos de Fábrega a solicitud del señor Jerónimo Ossa certifican que el día de la fecha el vapor Oroya, de la marina de guerra peruana fondeado en esta isla, ha estado trasbordando considerablemente cantidad de fondos de las lanchas americanas números 7 y 8 [...]" (Fondo Ministerio de Relaciones Exteriores, 1879, vol. 217, fojas 16-17).

12 Este buque fue comprado por el gobierno del Perú a la Pacific Steam Navigation Company (PSNC), firma inglesa de transporte, por más de 58 mil libras esterlinas. La firma del convenio fue en medio de la guerra y cinco días después del Combate Naval de Iquique y el de Punta Gruesa (Farcau, 2000).

\section{Si Somos Americanos. Revista de Estudios Transfronterizos}


Recién en septiembre de 1879, la máxima autoridad istmeña tomó cartas en el asunto respecto del tráfico de armas. ¿Qué ocurrió entonces? Desde que Valdés Vergara asumió su rol diplomático en Colombia, las demandas por el respeto de los tratados internacionales firmados entre Santiago y Bogotá fueron un imperativo en su labor. A pesar de la juventud del nacional - proveniente de una familia de estirpe política, dentro de los que destacan su hermano, Ismael, diputado por Santiago (1903-1906) y alcalde de esa ciudad (1912-1915); y su tío materno, José Francisco Vergara Etchevers ${ }^{13}$, fundador de Viña del Mar, comandante de la caballería chilena y uno de los personajes más importantes de la Guerra del Pacífico-, su gestión estuvo marcada por diversos hitos, entre los que cabe destacar, la intempestiva visita de los vapores peruanos y de bandera inglesa que prestaban servicios al Perú en Panamá y el apertrechamiento que estos hacían en los puertos del istmo con mercadería y materiales de guerra provenientes de Europa y Costa Rica.

En este contexto, que también se vinculó con el llamado período de las "Campañas marítimas" de la Guerra, la consigna era que quien tuviera el control del mar, ganaría el conflicto. Por eso, los diplomáticos en el Caribe vieron con recelo el tránsito de los vapores Talismán, Chalaco, Oroya y Limeña por Panamá, desde mayo hasta octubre de 1879 (Fondo Ministerio de Relaciones Exteriores, 1877-1886, Vol. 181, s.f.; 1879-1886, vol. 217, f. 20-60 y AMDIPLCH, 1879d, vol. 25). Asimismo, con la victoria en Angamos (captura del Huáscar, 8 de octubre de 1879), el dominio del mar estuvo asegurado y con ella se auguraba la victoria chilena sobre la entente peruano-boliviana (Fondo Ministerio de Relaciones Exteriores, 1879-1886, vol. 217, foja 32). A su vez, con ello, la importación de armas desde Panamá hacia los países en guerra con Chile adquirió una clara relevancia, puesto que después de la pérdida del poder naval (Burr, 1967, p. 148), Perú se vio obligado a recurrir a esta vía (el contrabando) y a ese método (préstamos y envío de armas desde Europa) para prolongar el conflicto, y con él, asegurar que Chile no anexara los territorios salitreros. Hubo mucha complicidad, según versan los razonamientos expresos en la correspondencia de Valdés Vergara y de Jiménez, de las autoridades de Panamá y de los administradores del puerto ${ }^{14}$.

13 Ministro de Guerra y Marina en el gobierno del presidente Aníbal Pinto, Ministro del Interior en el mandato de Santa María.

14 Ahora bien, la Cancillería nacional, al ver el lento trabajo del diplomático Antonio Jiménez, decidió remover a este de su cargo. Por esta razón se nombró, con fecha 23 de septiembre de 1879, a Ramón Rivera Jofré, cónsul general de la República en los Estados Unidos de Cauca, Magdalena, Bolívar y Panamá, con residencia en este último lugar (Fondo Misiones Diplomáticas, 1879c, vol. 25, s.f.). 
Las últimas actividades diplomáticas de Jiménez fueron la comunicación del arribo al istmo del vapor inglés Arequipa de la Pacific Steam Navigation Company a fines de octubre de 1879. Asimismo, consigna el diplomático, dentro de los pasajeros del Arequipa había unos comisionados peruanos que tenían como misión irse en el primer vapor que zarpara a Europa desde el puerto de Panamá, para comprar buques y elementos de guerra. España sería el principal destino, puesto que uno de los barcos en cuestión, la Numancia, figuraba dentro de la lista de prioridades (Fondo Ministerio de Relaciones Exteriores, 1879-1886, vol. 217, foja 33).

\section{Ocaso de 1879}

El 27 de octubre de 1879, en tanto, llegó desde Nueva York a la ciudad de Colón un vapor torpedo de 75 pies de largo por 9 de ancho y que navegaba a 20 millas por hora ${ }^{15}$. Según indica el cónsul Jiménez, esta embarcación iba a ser enviada a Perú. Por esta razón, el diplomático avisó inmediatamente al secretario del Estado de Panamá, José María Alemán, pidiéndole la detención y embargo de la nave (Fondo Ministerio de Relaciones Exteriores, 1879-1886, vol. 217, foja 44). No obstante, días más tarde, a principios de noviembre de 1879 (Fondo Ministerio de Relaciones Exteriores, 1879-1886, vol. 217, foja 50), el vapor inglés Colombia arribó desde Guayaquil con cuatro fuerzas de fierro lanzatorpedos, unos cincuenta o más cajones de materiales para el servicio de los marinos. Todos esos artículos fueron retornados, según instrucción del secretario del Istmo, por ser de contrabando de guerra ${ }^{16}$.

El vapor Alay se hallaba bajo la vigilancia y custodia del inspector y jefe del resguardo del puerto de Colón. Además, se había dado orden de impedir su salida, mientras no se declarara oficialmente la nacionalidad de la embarcación y la motivación que tenía

15 El vapor en cuestión es el Alay, una torpedera comprada a Inglaterra por el vicepresidente peruano José Francisco Canevaro, por un total de 9 mil libras esterlinas. Este hecho rompe la "neutralidad" de la potencia europea en el conflicto del Pacífico. La venta se efectuó a mediados de 1879. El despacho fue vía Atlántico hasta Nueva York y de ahí al puerto panameño de Colón. Ya en ese lugar, los distintos transportes peruanos y de la PSNC trataron de armar a esta embarcación. Para ello, realizaron, vía Guayaquil, diversos viajes con ese fin. Para mayores antecedentes, véase a Vicuña Mackenna (1881).

16 "Según comunicaciones que tengo de Guayaquil, indica Jiménez, aquellos materiales son partes constitutivas del servicio del vapor-torpedo que se encuentra actualmente detenido en esta agua, habiendo sido allí reconocidos por puertos muy competentes. Estando aquellos en los respectivos depósitos en este puerto, me permito pedir a Ud. en cumplimiento de mis deberes y de los de la neutralidad que corresponde a este estado, se sirve hacer llegar esta nota a conocimiento del señor presidente, a fin de que se dicte la respectiva orden de embargo de los artículos de contrabando de guerra que han traído de Guayaquil" (Fondo Ministerio de Relaciones Exteriores, vol. 217, foja 50).

\section{Si Somos Americanos. Revista de Estudios Transfronterizos}


esta de iniciar ruta hacia Callao (Fondo Ministerio de Relaciones Exteriores, 1879-1886, vol. 217, foja 52). Sin embargo, la medida adoptada por Alemán no fue del todo efectiva y dejó muchos vacíos, puesto que los oficiales de la aduana y del ferrocarril declararon que la información entregada por Jiménez era errónea, contraviniendo el libre tránsito de mercadería proveniente de Estados Unidos, lugar desde donde había sido remitido el Alay. Esto empantanó más las gestiones diplomáticas del cónsul de Chile.

Como solución, el gobierno chileno se dispuso a apresar esta nave, empleando para ello al transporte Amazonas, cuyo capitán era Manuel Thompson. La captura de este navío se produjo el 23 de diciembre de 1879, en el puerto de Ballenitas, Ecuador. Asimismo, en marzo de 1880, dicha torpedera fue anexada a la flota chilena y rebautizada como Guacolda. Además, con ese nombre participó en el bloqueo del Callao (Ahumada, 1982, pp. 7-8). Este hecho, por cierto, no pasó desapercibido. El periódico nacional de corte conservador y católico, El Independiente, señalaría al respecto:

La prensa ecuatoriana en masa ha protestado de este acto, calificándolo como un inaudito atropello de la soberanía de su país. Nuestros legistas y nuestros cucalones, por su parte, discuten el hecho acaloradamente, no faltando quienes opinen nuestro gobierno debe pagar el valor de la lancha como una satisfacción dada al Ecuador...

“[...] lo justo es dar la satisfacción, nosotros pediríamos que antes la exigiésemos de Colombia, por haber quebrantado de un modo vergonzoso y descarado, la neutralidad que estaba obligado a mantener en la Guerra del Pacífico" (Archivo Nacional de Colombia 1880-1911a).

En tanto, el ministro de Relaciones Exteriores, Miguel Luis Amunátegui, trató de informar los aciertos de su gestión a Valdés Vergara, así como también le otorgó el respaldo y la tranquilidad necesaria para resolver los inconvenientes en Colombia y Panamá. Otro de los hitos fue el control del Estrecho de Magallanes y la llegada de municiones traídas desde Europa por los vapores Glenelg, Genovese, Belle, Marranéese y el Hilton Castle (Fondo Misiones Diplomáticas, 1879c, vol. 25, s.f). Asimismo, el canciller señaló que a través del triunfo y las primeras maniobras de la "campaña de Tarapacá"17, el estado del Ejército chileno era "brillante" como el de la Escuadra.

17 La campaña de Tarapacá corresponde a la primera fase de las acciones terrestres planificadas por Chile durante la Guerra del Pacífico, durante noviembre y diciembre de 1879 (Bulnes, 1911; Vicuña Mackenna, 1880). 


\section{El incidente del transporte chileno Amazonas}

Al pasar de los días en los últimos meses de 1879, las evidencias y las pruebas en contra de la imparcialidad de Panamá en el conflicto del Pacífico eran cada vez más contundentes. Jiménez, en tanto, desde el istmo, enviaba noticias importantes a su superior en Colombia. Cabe señalar que las credenciales de su sucesor, Ramón Rivera, no fueron aceptadas de inmediato en Bogotá. Una de las informaciones relevantes relatadas por Jiménez, fue el arribo del transporte chileno Amazonas ${ }^{18}$ a tierras caribeñas.

Según lo que indicó el diplomático el 17 de octubre de 1879, los tripulantes del barco nacional, que venía de unas maniobras en el Estrecho de Magallanes ${ }^{19}$, desembarcaron desaforadamente en el puerto de Panamá. Bebieron licor a destajo y mantuvieron un ambiente festivo alrededor. En ese periplo, se dirigieron a una pulpería peruana, lugar en el que continuaron su juerga y en el que había un cuadro del Huáscar. El dueño del negocio los fastidió tanto con las hazañas y peripecias de la embarcación peruana, que uno de los marineros del Amazonas le rompió la pintura en la cabeza. Al sitio concurrió la policía panameña, produciéndose un pequeño altercado. Este hecho, que parece anecdótico, intensificó un sentimiento antichileno en el istmo y una clara simpatía por la causa peruana (Burr, 1967, pp. 147-150). El crisol de esta paradiplomacia fue el incidente del San Ramón del 8 de mayo de 1880, en el que fueron detenidos 11 ciudadanos chilenos acusados de piratería y de intentar abordar a la goleta ecuatoriana Enriqueta, navío que supuestamente llevaba armas a Perú (López, 2011).

18 Bulnes (1911) indica que el Amazonas partió a Panamá para dar caza al Oroya, que se encontraba en ese puerto (pp. 409-411).

19 El Amazonas, junto con la corbeta O'Higgins, habían emprendido viaje al Estrecho de Magallanes con el fin de evitar que la corbeta peruana Unión, capturara al vapor Genovese, que traía armas para Chile. Las instrucciones del ministro de Guerra y Marina de Chile, Rafael Sotomayor, en carta suscrita al comandante del Amazonas y del O'Higgins, el 2 de septiembre de 1879, era capturar a la Unión y proteger la llegada de la mercancía del Genovese. Lo anecdótico de todo fue que la Unión, que el 23 de julio 1879 había participado en la captura del vapor chileno Rímac, se dirigió hacia el Estrecho de Magallanes con el fin de evitar que el Glenelg, un vapor arrendado por Chile a Inglaterra, cumpliera su misión de proveer de pertrechos a Chile. En ese viaje, a mediados de agosto, capturó al Sakkarah, vapor de la compañía alemana Kosmos. Sin embargo, el capitán de la Unión, Aurelio García y García, no obtuvo ninguna información relevante de parte de los germanos, de esta forma, procedió a arribar a Punta Arenas. En ese lugar, se enteraron de que el Glenelg había pasado por la zona unas semanas antes. Ya en puerto, la Unión se proveyó de carbón, zarpando el 18 de agosto rumbo a Arica (Ahumada, 1982, pp. 32-33.)

\section{Si Somos Americanos. Revista de Estudios Transfronterizos}




\section{Panamá y Estados Unidos}

La demora de las comunicaciones entre los distintos representantes diplomáticos, a veces con varios días de retraso, afectaron la misión de Valdés Vergara en Colombia. Otro elemento que, sin duda, debe considerar un estudio más amplio del que se le ha dado en este trabajo, es el proyecto de construcción del canal en Panamá, que comunicará el océano Pacífico con el Atlántico, facilitando - en este caso- el comercio mundial. La obra, iniciada el 5 de julio de 1879 por la Compagnie Universelle du Canal Interocéanique de Panamá (Compañía Universal del Canal Interoceánico de Panamá), liderada por Ferdinand de Lesseps - un diplomático francés que años atrás había construido exitosamente, sin ser ingeniero, el Canal de Suez en Egipto-, provocó una verdadera revolución en el istmo. El ferrocarril de Panamá cambió de dueños en 1880), al pasar de manos norteamericanas a francesas durante la aventura de Lesseps. Sin embargo, la administración y los empleados eran, en su mayoría, panameños y norteamericanos.

Lo anterior significó para las aspiraciones hegemónicas de Estados Unidos el posicionamiento y eventual instalación de una potencia europea, como Francia, en el continente $^{20}$. Este hecho atentaba en contra la "Doctrina Monroe".

Los tratados internacionales firmados entre Panamá y Norteamérica ${ }^{21}$, como el Mallarino-Bidlack (1846) y el polémico Herrán-Cass (1857), aseguraban la primacía del país del norte en dicha zona y también en gran parte de Colombia, antigua Nueva Granada. Se puede concluir, a partir de las gestiones de Jiménez y Valdés en Panamá y Colombia, respectivamente, que los intereses independentistas de los istmeños se antepusieron a las reclamaciones chilenas por el tráfico de armas y la perpetuación de la neutralidad del Estado caribeño en la Guerra del Pacífico (Sosa y Arce, 1911). Además, la descentralización administrativa y política de Colombia y la libertad jurídica de Panamá, según

20 En 1839, el gobierno francés envió al ingeniero Napoleón Gazella a ver la factibilidad técnica para la construcción de un enlace entre la ciudad de Colón y Panamá. El estudio indicó que la geografía del sector impedía el anhelo europeo, como también de la República de la Nueva Granada (Araúz y Pizzurno, 1993).

21 Dentro de este esquema también entra el Tratado Clayton-Bulwer, convenido entre el secretario de Estado de Estados Unidos, John Clayton, y el ministro plenipotenciario de Inglaterra en Norteamérica, Henry Bulwer, el 19 de abril de 1850, para neutralizar sus influencias, además de impedir que dichos países pudieran colonizar o controlar alguna nación de Centroamérica. En esta misma lógica, es posible incluir las Proposiciones (tratado) Webster-Crampton, firmado por el secretario de Estado norteamericano, Daniel Webster, y el ministro plenipotenciario inglés en EE.UU., Sir John Fiennes Twisleton Crampton, el 30 de abril de 1852. Este convenio fue una serie de proposiciones a Nicaragua y Costa Rica para la resolución de sus conflictos limítrofes. Tanto EE.UU. como el Reino Unido tenían intenciones de abrir un paso interoceánico en América Central. De ahí, entonces, la preocupación de ambas potencias (Brockway, 1958; Bunster, 1977; Haskin, 1914). 
consta en la Constitución de 1873, no ayudaron a que se consolidara la misión JiménezValdés. La atomización de la Gran Colombia durante los años treinta aún ronda dentro del espíritu de los liberales y separatistas panameños (Araúz y Pizzurno, 1993; Gasteazoro, Aráuz y Múñoz, 1980).

El rol de Norteamérica, en este caso, vino a ser el apoyo técnico, político y militar que necesitaban los istmeños para lograr su objetivo independentista. En el contexto del conflicto del Pacífico, el equilibrio de poder en Sudamérica estuvo en una situación crítica. Las victorias chilenas durante 1879-1880, tanto en las campañas terrestres como en las marítimas, hicieron prever, en un escenario hipotético, la asunción de Chile como una potencia regional. Los triunfos nacionales significaron - a la postre - según interpretaron sus vecinos del continente y algunas potencias foráneas, la posibilidad de anexionar los terrenos en disputa, el aumento del poder naval y militar, y la administración chilena del dinero y capital salitrero en juego, entre otros ${ }^{22}$. Como corolario, cabe mencionar que la misión de Newton Pettis, realizada —en agosto de 1879- a título personal por el ministro plenipotenciario de Norteamérica en Bolivia, fue el primer intento de "arbitraje" e interposición del país del norte en la guerra (Bulnes, 1911, pp. 423-426). Un año más tarde, el secretario de Estado, William Evarts, instruyó a los representantes en Lima, La Paz y Santiago para ofrecer "buenos oficios" por parte de EE.UU. (Ahumada, 1982, VIIVIII, p. 58). De ahí, entonces, la antipatía hacia Estados Unidos y sus aliados americanos (Sater, 1986, 1990; y Burr, 1967).

\section{Conclusiones}

Las relaciones diplomáticas entre Chile y la Unión Colombiana durante 1879 no fueron las mejores. El factor Panamá fue siempre un tema álgido, tanto para los nacionales como para los caribeños. En el contexto de la guerra, la participación del liberal Domingo Santa María González como ministro de Relaciones Exteriores y Colonización, desde el 17 de abril hasta el 20 de agosto de 1879, se caracterizó por la construcción de una imagen país muy pétrea, pero asequible al diálogo. Santa María, un político de carácter, creyó que Chile, en la empresa de la guerra, tenía mucha ventaja por sobre Bolivia; no obstante, con Perú, estimaba, había una igualdad en términos militares y navales. Por una parte, las demás naciones americanas, durante el inicio de las hostilidades entre Chile y

22 Informes inéditos de diplomáticos extranjeros durante la Guerra del Pacífico. Santiago de Chile: Andrés Bello, 1980.

\section{Si Somos Americanos. Revista de Estudios Transfronterizos}


Bolivia, pensaron que la salida diplomática y la figura de la mediación, eran dos herramientas que podían evitar el derramamiento de sangre entre países hermanos.

Por otra parte, durante el principio del segundo año de la Guerra del Pacífico, las sendas victorias chilenas en la campaña marítima, tras el triunfo de Angamos, y terrestre de Tarapacá, provocaron en la población un inusual optimismo y un discurso hegemónico nacionalista. Tras la ocupación de la provincia de Tarapacá por parte de los chilenos, el general Mariano Prado, presidente del Perú (1876-1879), decidió partir a Europa para comprar armamento. En su lugar, dejó al vetusto vicepresidente Luis La Puerta ${ }^{23}$. La poca capacidad de mando de La Puerta, sumado al inclemente desconcierto del pueblo peruano por las recientes derrotas y al surgimiento de caudillos, los comandantes Argüidas, Iglesias y Piérola se rebelaron en contra del gobierno establecido, generando una revolución popular. Así, pues, el 23 de diciembre de 1879, Piérola entró triunfante a Lima, donde se autoproclamo "dictador" (Bulnes, 1911, pp. 410-415). Una situación similar se generó en Bolivia, donde el mandatario Hilarión Daza fue depuesto por el general Narciso Campero.

Las noticias de la deposición de Prado fueron ampliamente comentadas por los representantes chilenos y colombianos. El cónsul general de ese país en Chile, Ricardo de Francisco, indicó lo siguiente:

La prensa chilena y la cámara legislativa se ocupan con tesoro de algunas de las condiciones que deben imponerse a los aliados, a quienes generalmente se considera vencido, y casi unánimemente se aboga porque el territorio de Tarapacá, tan rico en huaneras y salitreras, se incorpora a Chile y se conserva por él para siempre a título de propiedad. Algunos menos entusiastas dicen que la incorporación tiene que hacerse, pero que el Congreso no debe aún decretarla (Archivo Nacional de Colombia 18801911b, foja 191).

En efecto, tras la ocupación de Tarapacá, Chile tomó el control de las guaneras y salitreras de la zona. Las victorias chilenas no eran una preocupación para los colombianos ni para el resto de países que observaban el desarrollo de la guerra. La atención estaba centrada en la posible anexión de las tierras en disputa por parte de Chile.

23 "Por cuanto estoy autorizado para salir del país, por la Resolución Legislativa de 9 de mayo de 1879, y asuntos muy importantes y urgentes demandan mi presencia en el extranjero, y es mi deber y mi deseo hacer cuanto pueda a favor del país; decreto: Artículo Único. Encárguese de la Presidencia de la República S. E. el Vicepresidente, conforme a los artículos 90 y 93 de la Constitución. Imprímase, publíquese y circúlese para su debido cumplimiento. Dado en la Casa de Gobierno en Lima, a 18 de diciembre de 1879”. Firma Mariano Ignacio Prado. 
Durante 1879, la misión diplomática de Chile en Colombia fue impedir el tráfico de armas por el istmo, pero además, en este intento se debía tratar de hacer valer el Tratado Mosquera-Irarrázaval de 1844 y la modificación/actualización de 1853, que garantizaba una neutralidad expresa en caso de guerra. Obviamente, Trujillo, presidente de la unión colombiana, fijó el 2 de junio de 1879 — debido a la simpatía que tenía con la causa peruana y con los negocios que se auguraban tras la conferencia de París, en la que se decidió la construcción del Canal- al istmo como una zona franca, de tránsito, sin mediar en disputas o controversias de derecho internacional público. Esto - en la lógica del mandatario colombiano - era una muestra de civilización y cultura, y un mejor escenario que un conflicto armado entre naciones hermanas. Valdés Vergara, en conjunto con el cónsul nacional en Panamá, impusieron severas críticas a la gestión de Trujillo, enviándole continuamente misivas al canciller de la Unión. Tras la captura del Huáscar y el éxito de las campañas terrestres de Tarapacá, Tacna y Arica, la ofensiva peruana tuvo que utilizar con mayor ahínco la ruta del istmo. Esto para asegurar el apertrechamiento y para generar en otros países del continente, una creciente antipatía por la causa chilena. Ante la amenaza de que se trasladara el conflicto al istmo, Valdés Vergara solicitó al gobierno de Chile que el transporte Amazonas ${ }^{24}$ se dirigiera a Panamá para frenar y amedrentar la afluencia de vapores, buques y goletas que visitaban esa zona en busca de pertrechos para el Perú. Así, pues, la disputa diplomática tomó otro cariz.

\section{Referencias}

Ahumada, P. (1982). Guerra del Pacífico. Documentos Oficiales, Correspondencias y demás publicaciones referentes a la guerra, que ha dado a luz la prensa de Chile, Perú y Bolivia. Santiago, Chile: Editorial Andrés Bello. 8 vol. (1884).

Araúz, C., y Pizzurno, P. (1993). El Panamá colombiano (1821-1903). Panamá: Banco de Ahoros y La Prensa.

Archivo General del Ministerio de Relaciones Exteriores de Chile. (1879-1881). Copiador de correspondencia enviada por el Ministerio de RR.EE. de Chile a los Agentes Diplomáticos de Chile en América y Europa. Vol. 62A, fojas 12-13.

24 El historiador Gonzalo Bulnes (1961) señala que la gestión de Valdés Vergara en Colombia, se caracterizó por ser muy clara y directa. De hecho, denunció en reiteradas ocasiones el trasporte de armas por el istmo de Panamá, Estado Soberano de la Unión Colombiana. Asimismo, según Rivas, el representante nacional calificó al país caribeño como "un depósito de armas para el Perú" (p. 497).

\section{Si Somos Americanos. Revista de Estudios Transfronterizos}


Archivo Nacional de Colombia. (1880-1911a). Diplomática y consular. Consulado de Colombia en Chile. Carta de Ricardo de Francisco a Luis Carlos Rico, 28 de enero de 1880.

Archivo Nacional de Colombia. (1880-1911b). Diplomática y consular consulado de Colombia en Chile 1880-1911. Carta del Cónsul General de los Estados Unidos de Colombia, Ricardo de Francisco, al Secretario de lo Interior y Relaciones Exteriores de la Unión, Luis Carlos Rico, 1 de enero de 1880, foja 191.

Arteaga, M., y Arteaga, J. (1986). Historia política de Colombia, Volumen 4. Bogotá, Colombia: Intermedio-El Tiempo.

Barbé, E. (1987). El equilibrio del poder en la Teoría de las Relaciones Internacionales. Revista Afers Internacionals 11.

Barros, D. (1914). Historia de la Guerra del Pacifico. Obras completas. Santiago, Chile: Imprenta, Litografía y Encuadernación Barcelona.

Barros, M. (1990). Historia diplomática de Chile, 1541-1938. Santiago, Chile: Andrés Bello.

Bizzarro, S. (2005). Historical dictionary of Chile. Tercera Edición, Scarecrow Press.

Boletín de la Guerra del Pacífico. (1979). Santiago, Chile: Andrés Bello.

Bonilla, H. (1979). La dimensión internacional de la Guerra del Pacífico. Desarrollo Económico 19 (73).

Brockway, T. (1958). Documentos básicos de la política exterior estadounidense. [Traducción de Mario A. Marino]. 1a. Edición. Buenos Aires, Argentina: Ágora.

Bulnes, G. (1911). Guerra del Pacífico. De Antofagasta a Tarapacá. Tomo I. Valparaíso, Chile: Sociedad Imprenta y Litografía Universo.

Bulnes, G. 1914. La Guerra del Pacífico. De Tarapacá a Lima. Vol. II. Valparaíso, Chile: Sociedad Imprenta y Litografía Universo.

Bunster, E. (1977). Crónicas del Pacífico. Santiago, Chile: Andrés Bello.

Burr, R. (1957). El equilibro de poder en el siglo XIX en Sudamérica. Clío 28, 5-39. 
Burr, R. (1967). By the reason or force. Chile and the balancing of power in South America. 1830-1905. Los Angeles, California: University of California Press.

Collier, S. (2005). Chile la construcción de una república 1830-1865: política e ideas. Santiago, Chile: Pontificia Universidad Católica de Chile.

De Plaza, J. (1850). Apéndice a la recopilación de Leyes de la Nueva Granada, formado y publicado por orden del Poder Ejecutivo, don José Antonio de Plaza. Contiene toda la legislación nacional vijente desde 1845 hasta 1849 inclusive. Bogotá, Colombia: Imprenta del Neo-Granadino.

Farcau, B. (2000). The Ten Cents War: Chile, Peru, and Bolivia in the War of the Pacific, 18791884. Westport, Connecticut: Praeger.

Fermandois, J. (2005). Mundo y fin de mundo. Chile en la política mundial. 1900-2004. Santiago, Chile: Pontificia Universidad Católica de Chile.

Fondo Ministerio de Relaciones Exteriores. (1877-1886). Gobierno y Agentes Diplomáticos de Colombia en Chile. Volumen 181.

Fondo Ministerio de Relaciones Exteriores. (1879-1886). Notas de los Consulados de Chile en Panamá. Volumen 217.

Fondo Misiones Diplomáticas. (1879a). Misiones Diplomáticas de Chile en el Extranjero. Oficios recibidos por la Legación de Chile en Colombia. Vol. 25, s.f.

Fondo Misiones Diplomáticas. (1879b). Oficio enviado por el Cónsul General de Dinamarca en Colombia al representante chileno Francisco Valdés Vergara, 17 de septiembre de 1879 y la carta remitida por el Plenipotenciario del Emperador de Alemania en el país caribeño al encargado de negocios Valdés Vergara, 3 de septiembre de 1879. Vol. 25, s.f.

Fondo Misiones Diplomáticas. (1879c). Carta de Amunátegui a Valdés Vergara, 28 de noviembre de 1879. Vol. 25, s.f.

Fondo Misiones Diplomáticas. (1879d). Correspondencia de mayo a noviembre de 1879. Vol. 25. 
Gasteazoro, C., Aráuz, C., y Muñoz, A. (1980). La historia de Panamá en sus textos. Tomo I. Panamá: Universitaria.

Góngora, M. (2006). Ensayo histórico sobre la noción de estado en Chile en los siglos XIX y XX. Santiago, Chile: Universitaria.

Haskin, F. J. (1914). The Panamá Canal. New York: Double Day, Page \& Company.

Hilton, S. (2001). Los nuevos Estados americanos en el sistema internacional contemporáneo. 1775-1895. En Pereira, J. (coord.), Historia de las Relaciones Internacionales (pp. 5974). Barcelona, España: Ariel.

Informes inéditos de diplomáticos extranjeros durante la Guerra del Pacífico. 1980. Santiago, Chile: Andrés Bello.

Jara, M., y López, F. (2012). Guerra y Diplomacia: La Legación chilena en los Estados Unidos de Colombia (1879-1880). En A. Sánchez y A. Delgado, Los nuevos Estados-nación y su inserción en el contexto internacional 1820-1903 (pp. 415-443). Morelia, México: Instituto de Investigaciones Históricas de la Universidad Michoacana de México e Institut des Langues et des Cultures d'Europe et d'Amérique (Ilcea).

López, F. (2011). Análisis documental y epistorlar de la Legación de Chile en los Estados Unidos de Colombia durante los primeros años de la Guerra del Pacífico (1879-1880). Tesis para optar al grado de Licenciatura en Historia, Universidad Católica de la Santísima Concepción.

Memorias del Ministerio de Relaciones Exteriores de Chile, años 1879, 1880.

Meneses, E. (1986). Los límites del Equilibrio de Poder: La política exterior chilena a fines del siglo pasado, 1891 1902. Revista Opciones 9, 89-118.

Meneses, E. (1989). El factor naval en las relaciones entre Chile y los Estados Unidos (18811951). Santiago, Chile: Ediciones Pedagógicas Chilenas S.A.

Neila, J. (2008). El Equilibrio de Poder. En J. Pereira (coord.), Diccionario de Relaciones Internacionales Política Exterior. Barcelona, España: Ariel.

Pereira, J. C. (coord.). (2008). Diccionario de Relaciones Internacionales Politica Exterior. Barcelona, España: Ariel. 
Pereira, J. C. (coord.). (2009). El estudio de la sociedad contemporánea actual. En Pereira, J.C. (coord.), Historia de las Relaciones Internacionales (pp. 37-62). Barcelona, España: Ariel.

Rivas, R. (1961). Historia diplomática de Colombia (1810-1934). Bogotá, Colombia: Ministerio de Relaciones Exteriores.

Rodríguez-Arenas, F. (2006). Bibliografía de la literatura colombiana del siglo XIX. Tomo I, $A-L$. Buenos Aires, Argentina: Stockcero.

Rubilar, M. (2008). Guerra y Diplomacia: Las relaciones chileno-colombianas durante la Guera y Posguerra del Pacífico. 1879-1886. Tzin-Tzun 42, 49-86.

Rubilar, M. (2010). Guerra, diplomacia y poder naval: Las relaciones internacionales de Chile, Colombia y Estados Unidos durante la guerra y posguerra del Pacífico, 1879-1886. Suficiencia investigadora para optar al Diplomado de Estudios Avanzado (D.E.A.) del departamento de Historia moderna, contemporánea, de América, periodismo y comunicación audiovisual y publicidad, Universidad de Valladolid. Material inédito.

Salazar, G. (2005). La construcción de Estado en Chile: (1760-1860). Democracia de los pueblos, Militarismo ciudadano, Golpismo oligárquico. Santiago, Chile: Editorial Sudamericana.

Sater, W. (1981). La agricultura chilena y la Guerra del Pacífico. Historia 16, 125-149.

Sater, W. (1986). Chile and the War of the Pacific. Lincoln, Nebraska and London, UK: University of Nebraska Press.

Sater, W. (1990). Chile and the United States: Empires in conflict. Athens (Georgia) and London, UK: The University of Georgia Press.

Sater, W. (2007). Andean Tragedy: Fighting the War of the Pacific, 1879-1884. Lincoln: University of Nebraska Press.

Sosa, J., y Arce, E. (1911). Compendio de historia de Panamá. Panamá: Editorial Diario de Panamá.

Souler, R. (1987). Idea y cuestión nacional y latinoamericana. De la independencia a la emergencia del liberalismo. México: Siglo XXI.

122 Si Somos Americanos. Revista de Estudios Transfronterizos 
Volumen XIV / No 1 / enero-junio 2014 / pp. 101-123

Souler, R. (1999). La invasión de Estados Unidos a Panamá. Neocolonialismo en la posguerra fría. México: Siglo XXI.

Vicuña Mackenna, B. (1880). Historia de la Campaña de Tarapacá, Tomos I y II. Santiago, Chile: Imprenta Cervantes.

Vicuña Mackenna, B. (1881). Historia de la Campaña de Lima. Santiago, Chile: Cervantes. 